\title{
Uterine Incision at the Fundus, Transitory Devascularisation, and Myometrial Resection for Uterine Preservation in Anterior Accretism: A Case Report
}

\author{
Authors: \\ Rayra Amana Macêdo Maciel, ${ }^{1}{ }^{*}$ Caio Ribeiro Vieira Leal, ${ }^{1}$ Bárbara \\ Flecha D’Abreu, ${ }^{1}$ Mário Dias Corrêa Júnior, ${ }^{1,2}$ Admário Silva Santos \\ Filho ${ }^{1}$ \\ 1. Department of Gynaecology and Obstetrics, Hospital das Clínicas, Federal \\ University of Minas Gerais (UFMG), Belo Horizonte, Brazil \\ 2. Department of Gynaecology and Obstetrics, Federal University of Minas Gerais \\ (UFMG) School of Medicine, Belo Horizonte, Brazil \\ *Correspondence to lealcaiorv@gmail.com
}

Disclosure: The authors have declared no conflicts of interest.

Received: $\quad 16.01 .21$

Accepted: $\quad 24.03 .21$

Keywords: $\quad$ Caesarean section (C-section), placenta accreta spectrum (PAS), postpartum haemorrhage.

Citation:

EMJ Repro Health. 2021;7[1]:54-59.

\section{Abstract}

Placenta accreta spectrum is a serious obstetric condition related to abnormal adherence of placental tissue to the myometrium and high maternal and fetal morbidity. In order to achieve the best outcome, the management of this condition must be carried out by an experienced multidisciplinary team and the individual characteristics of the patient must be taken into consideration, such as comorbidities and desire for reproductive preservation. This case report presents the conservative surgical management of placenta accreta spectrum in a 23-year-old patient who underwent an elective caesarean section with uterine preservation because of anterior placenta increta. The authors performed a transverse uterine incision at the fundus with transitory uterine devascularisation of the lower uterine segment with partial myometrial removal. This technique was successful for controlling the haemorrhage and preserving the uterus, with no complications.

\section{INTRODUCTION}

Placenta accreta spectrum (PAS) is a set of obstetric conditions with abnormal adhesion of placental tissue to the myometrium. These conditions differ according to the degree of trophoblastic invasion to the myometrium: attachment to the myometrium (placenta accreta), invasion into the myometrium layer

(placenta increta), or invasion through the myometrium to reach the serosa and other adjacent structures (placenta percreta). ${ }^{1}$ PAS is associated with high maternal and fetal morbidity and mortality, particularly because of the risk of major haemorrhage, requirement of haemotransfusion, peripartum hysterectomy, and prematurity. ${ }^{2}$ The haemorrhage is related to the depth of myometrial invasion, the size of the 
adhesion area, and the presence of invasion of adjacent structures by the placenta. ${ }^{3}$

In recent years, the most common interventional approach for PAS has been elective caesarean section (C-section) followed by hysterectomy; ${ }^{3}$ however, conservative interventions can also be used, while taking into account the reduction of unfavourable outcomes, such as bleeding and the subsequent complications. ${ }^{4}$ In this article, the authors report a case of a patient with anterior placenta increta who underwent elective C-section with uterine preservation. The technique applied was uterine incision at the fundus, with transitory uterine devascularisation with partial myometrial removal.

\section{CASE REPORT}

A 23-year-old patient, gravida 2, para 1 (one previous C-section), with no comorbidities, was monitored in a low-risk prenatal care setting from 11 weeks' gestation. She had no history of any surgical procedures (apart from the C-section), uterine manipulation, smoking, or previous pelvic infection.

The patient arrived at the maternity ward emergency room of a high-complexity hospital at 38/39 weeks' gestation (dated from the last menstrual period, accordant with the first ultrasound at 11/12 weeks), presenting prior obstetric ultrasonography results performed in an outpatient service, which indicated an anterior increta placenta. New ultrasonography (transabdominal and endovaginal) was performed by specialists. The results showed an anterior placenta with low segmental insertion (and not reaching the internal orifice of the uterine cervix). It also revealed, on the right area of low segment, a partial loss of myometrial continuity, increased vascularisation on Doppler ultrasound findings, and a healthy fetus.

On examination of the patient's medical records from prenatal care, it became apparent that a diagnosis of anterior placenta accreta was already suspected from the first performed ultrasound. This detailed a placenta with anterior topical insertion, presenting inaccurate limits and irregularities with the myometrium and prolongations of its basal vessels penetrating into the uterine wall using Doppler. In another ultrasound performed at 12/13 weeks' gestation, a placenta of anterior insertion was reported, which covered the internal orifice of the cervix, without other changes. The next ultrasound was performed at $31 / 32$ weeks' gestation and the abnormality was neither described nor investigated.

The patient was only advised about the diagnosis when entering the high-complexity hospital. The patient expressed her desire for future pregnancy, which led the surgical plan to attempt a conservative technique. The patient was informed about the higher risk of uterine rupture if future pregnancy was to occur after the procedure, in addition to the risk of major haemorrhage and the possibility of having to perform a hysterectomy during surgery. A multidisciplinary team constituting gynaecological surgeons, obstetricians, anaesthesiologists, and neonatologists were responsible for the surgical plan. In the surgical plan, the team considered how the ultrasound scans suggested placenta increta in the right lower uterine segment, with no signs of other organ involvement and an absence of signs of praevia or low-lying placenta. It was planned to perform fetal extraction through a transverse incision in the uterine fundus. In order to reduce the risk of haemorrhage, the use of a Penrose drain for uterine reversible devascularisation was considered. The resection of the area of placental invasion would be performed in the absence of intra-operative signs of placenta percreta, from the exposed myometrium with the transverse incision. Laboratory tests were checked, as well as blood components, and an intensive care unit was reserved because of the high risk of major haemorrhage.

Pre-operative haemotransfusion was performed because the patient had a haemoglobin count of $8.5 \mathrm{mg} / \mathrm{dL}$. The procedure occurred under spinal anaesthesia with bladder catheterisation. The anaesthesia team was prepared to alter the procedure to general anaesthesia in case of haemodynamic instability. A median xipho-pubic incision was chosen for better uterine exposure and exteriorisation because of advanced gestational age and the risk of requiring extensive adhesiolysis. The upper placental border was identified by palpation and a unique transverse incision at $3 \mathrm{~cm}$ from the uterine fundus was performed. The fetal extraction was pelvic, with a first-minute Apgar score of 9. A Penrose drain 
was used for tying the uterine segment, including the uterine and ovarian arteries with reversible and transitory uterine devascularisation (Figure 1). After the placenta was extracted, placenta increta was visible in the uterine anterior wall; the uterine segment showed invasion of $<70 \%$ of myometrial layer from gross visual inspection.

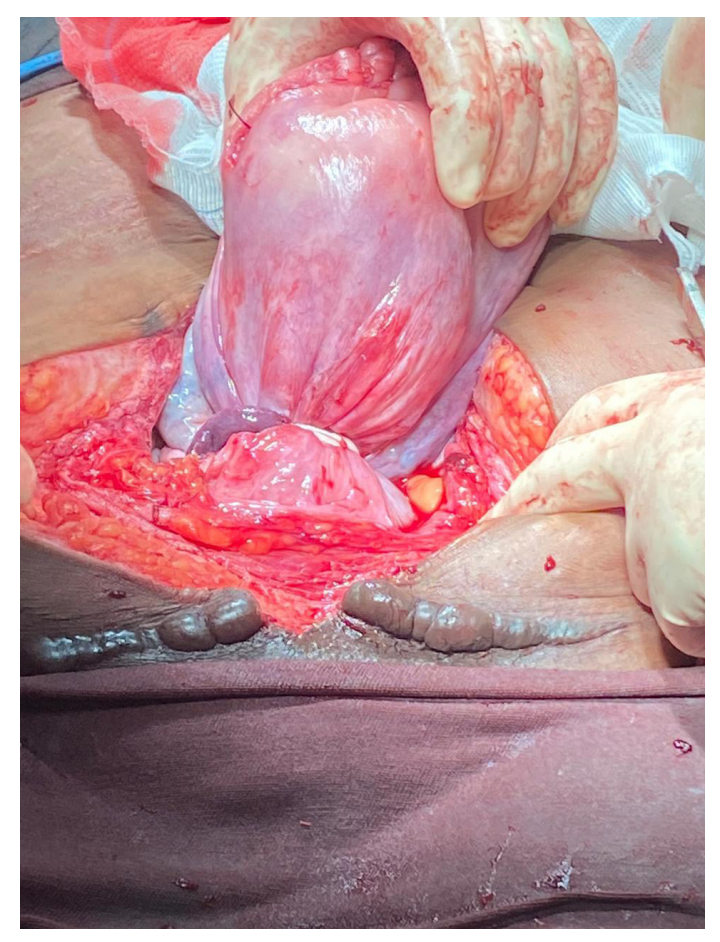

Figure 1: Penrose drain reducing vascular flow in uterine and ovarian arteries.

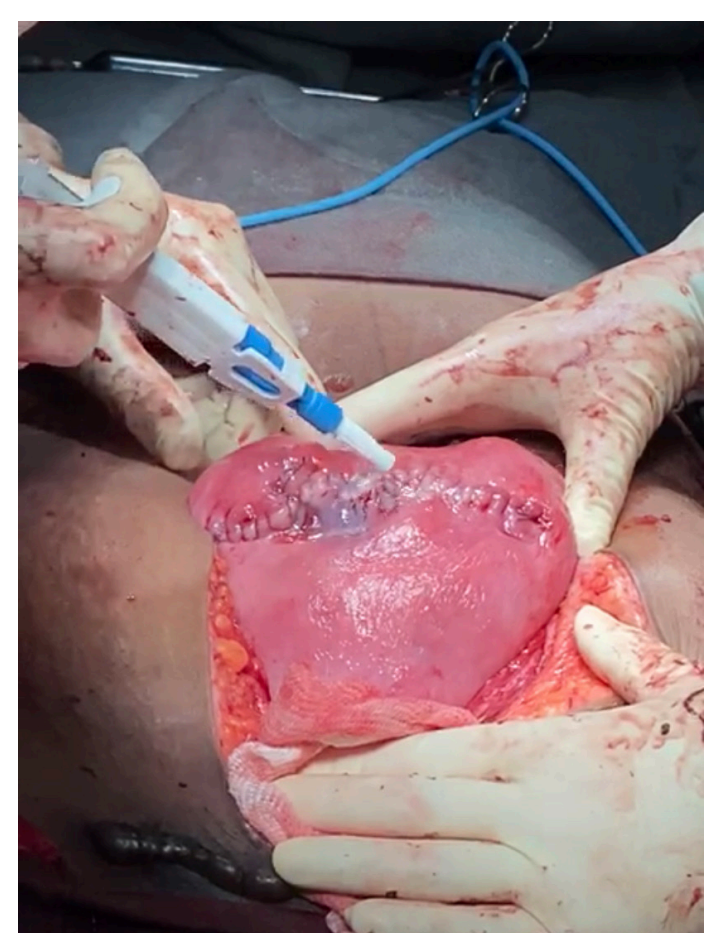

Figure 2: Application of fibrin glue on two-plane hysterorrhaphy. 


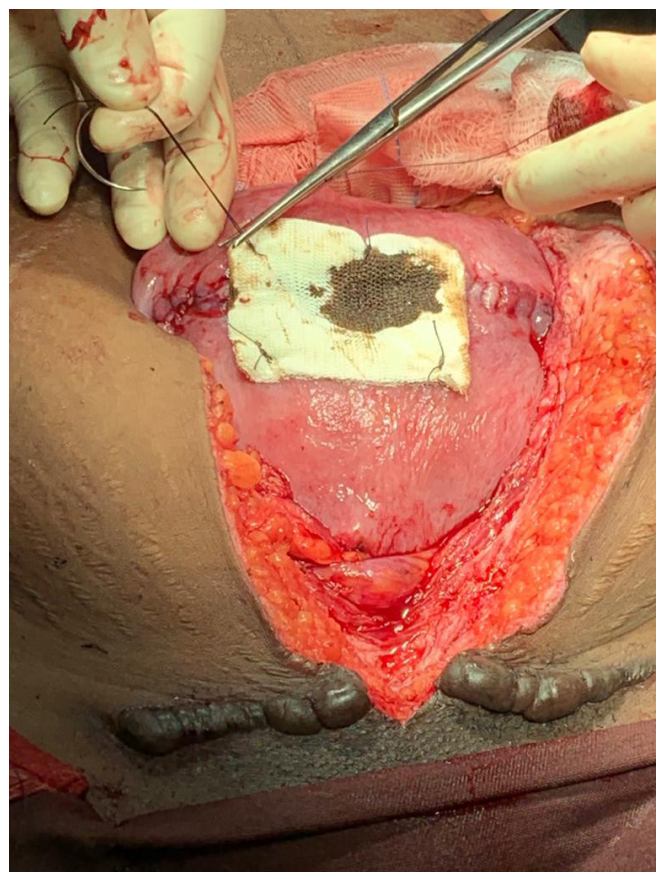

Figure 3: Application of oxidised regenerated cellulose.

Longitudinal resection of the myometrial area with placental invasion was performed: about $10 \mathrm{~cm}$ longitudinally and $4 \mathrm{~cm}$ vertically, covering the entire thickness of the uterine wall. It was sutured for internal recomposition of the uterine segment and hysterorrhaphy, in a double-layer suture. The uterine segment was untied and uterine revascularisation was observed. Haemostasis was reviewed, followed by application of fibrin glue and oxidised regenerated cellulose (absorbable haemostat) over the hysterorrhaphy (Figures 2 and 3). There were no signs of placental invasion on bladder or ureteral lesions. The patient was stable and did not require further haemotransfusion or intensive care. She was referred to the obstetrics and gynaecology ward for clinical follow-up; here, she was discharged after 72 hours and referred for outpatient follow-up. She was advised about the need for high-risk prenatal follow-up in the case of subsequent pregnancies, as well as the possibility of recurrence of placenta accreta and uterine rupture.

\section{DISCUSSION}

Although the pathophysiology of PAS is not completely understood, it is known to be related to the abnormal decidualisation process, with abnormal placental invasion and adherence within the uterine scar tissue as a result of an endometrial-myometrial interface defect. ${ }^{5}$ The two main risk factors are previous $\mathrm{C}$-section and placenta praevia in current pregnancy, ${ }^{6}$ and the risk is increased when both factors are present ${ }^{1}$ or by a higher number of C-sections. ${ }^{7}$ PAS has an estimated prevalence of between $0.01 \%$ and $1.1 \%$ in the general population ${ }^{8}$ and there has been a significant increase in its incidence in the last 40 years, mainly as a result of high C-section rates.

Approach to PAS should be performed by an experienced multidisciplinary team, preferably through an elective C-section or hysterectomy, between 34 weeks and 35 weeks and 6 days of gestational age (degree 1A of evidence). ${ }^{1}$ If possible, the diagnosis should be made in the prenatal stage by ultrasonography or MRI in order to allow for adequate planning., ${ }^{1,9,10}$ Some image findings (ultrasound or MRI) have been related to PAS, such as multiple placental lacunae (intraplacental sonolucent spaces), loss of the hypoechoic area behind the placenta, loss of the line representing the bladder wall-uterine serosa, myometrial thinning, abnormal vascularity, and uterine bulging into the bladder." The conservative strategy includes procedures that 
aim for uterine preservation, not only owing to the risks of a peripartum hysterectomy but also for the desire to preserve fertility. These conservative approaches essentially leave the placenta in situ to wait resorption, which should occur between 4 weeks and 12 months, and resect the invaded tissue with the placenta.4,12 However, potential complications include infection, intra-operative bleeding, and the need for hysterectomy.,12,13 Adjuvant treatment with methotrexate is not recommended because of adverse effects. ${ }^{4,12}$

Since the report of the 'one-step conservative surgery' for placenta accrete in 2004, which consists of resection of invaded tissue and myometrial reconstruction, other approaches have been described for bleeding control, including vascular interruption of newly formed vessels, bilateral uterine artery ligation, and iliac balloon insufflation. Resection of the area of placental accretism with myometrial repair is considered appropriate when the anterior defect involves up to $50 \%$ of the uterine circumference. Fibrin glue and oxidised cellulose are useful in the control of bleeding and haemostasis. ${ }^{14}$

In 2019, a study was published that described and evaluated the technique of parallel transverse uterine incisions, which consists of a transverse incision at the uterine fundus for fetal extraction and a segmental transverse incision above the upper border of the placenta for resection of the invaded myometrium. This is followed by pelvic devascularisation by tourniquet with two rubber tubes: the first is tightened to the lower uterine segment to restrict uterine flow and the second is connected to the uterine body to restrict ovarian flow, along with insufflation of occlusion balloons in internal iliac arteries. The study showed significant statistical differences regarding blood loss, need for haemotransfusion, conversion to hysterectomy, and admission to the intensive care unit. ${ }^{13}$

The technique of single uterine incision at the fundus with transitory uterine devascularisation using the Penrose drain, tightly ligating the lower uterine segment and ovaries for concomitant compression of uterine and ovarian arteries, represents a promising adaptation of the myometrial resection and reconstruction techniques. The technique is efficient and allows for bleeding control with less-complex surgical techniques. The single transverse uterine incision at the fundus reduces bleeding foci and provides more time to control bleeding in emergency situations, including ligation of uterine arteries or insufflation of pelvic vascular balloons, as in the case of intra-operative diagnosis of PAS. In addition, a single transverse incision provides fewer foci of fragility in the uterine wall, which likely reduces the risk of uterine rupture in subsequent pregnancies compared to techniques in which more than one incision is performed.

Proper planning and appropriate patient selection are essential. The technique can be used on patients with anterior placenta accreta, in which the area of accretism does not compromise $>50 \%$ of the uterine circumference, in addition to the absence of signs of placenta percreta. All patients should be thoroughly informed about the intra-operative risks of the procedure, in addition to the risks of uterine rupture in case of new pregnancy. The team must be prepared for potential complications and the possibility of switching from the conservative procedure to an obstetric hysterectomy.

\section{CONCLUSION}

Although hysterectomy is the standard treatment for PAS in many countries, ${ }^{12}$ conservative techniques, especially accretism resection with myometrial repair, are excellent options for selected cases. The choice of treatment should consider not only the surgeon and patient's preference but also the resources and circumstances of the approach. The authors advise that all patients who are candidates for the procedure of uterine incision at the fundus with transitory uterine devascularisation or other conservative techniques should be thoroughly informed regarding the risks of the procedure. These include risk of major intra-operative haemorrhage and the risk of recurrence of placenta accreta, in addition to higher risk of uterine rupture by conceiving a subsequent pregnancy in a uterus that has had the lower uterine segment and part of the anterior uterine wall with myometrium approaching the uterine fundus removed. Prospective studies with multiple cases of the uterine incision at the fundus, with transitory uterine devascularisation with myometrial resection, are important to evaluate the applicability and to compare with other techniques already consolidated. 


\section{References}

1. Society of Gynecologic Oncology; American College of Obstetricians and Gynecologists and the Society for Maternal-Fetal Medicine; Cahill AG et al. Placenta accreta spectrum. Am J Obstet Gynecol. 2018;219(6):B2-16

2. Balayla J, Bondarenko HD. Placenta accreta and the risk of adverse maternal and neonatal outcomes. J Perinat Med. 2013;41(2):141-9.

3. Einerson BD, Branch DW. Surgica management of placenta accreta spectrum. Clin Obstet Gynecol. 2018;61(4):774-82.

4. Sentilhes $L$ et al. Conservative management of placenta accreta spectrum. Clin Obstet Gynecol. 2018;61(4):783-94

5. Jauniaux E, Burton GJ.

Pathophysiology of placenta accreta spectrum disorders: a review of current findings. Clin Obstet Gynecol. 2018;61(4):743-54.

6. Carusi DA. The placenta accreta spectrum: epidemiology and risk factors. Clin Obstet Gynecol 2018;61(4):733-42

7. De Mucio B et al. A systematic review and meta-analysis of cesarean delivery and other uterine surgery as risk factors for placenta accreta. Int J Gynecol Obstet. 2019;147(3):281-91.

8. Jauniaux $\mathrm{E}$ et al. Prevalence and main outcomes of placenta accreta spectrum: a systematic review and meta-analysis. Am J Obstet Gynecol. 2019;221(3):208-18.

9. Erfani $\mathrm{H}$ et al. Maternal outcomes in unexpected placenta accreta spectrum disorders: single-center experience with a multidisciplinary team. Am J Obstet Gynecol. 2019;221(4):337.e1-5.

10. Palacios-Jaraquemada JM et al. Systematic review on near miss cases of placenta accreta spectrum disorders: correlation with invasion topography, prenatal imaging, and surgical outcome. J Matern Feta Neonatal Med. 2020;33(19):3377-84.
11. Silver RM. Clinical features and diagnosis of placenta accreta spectrum (placenta accreta, increta, and percreta). 2021. Available at: https://www.uptodate.com/ contents/6759. Last accessed: 29 March 2021.

12. Palacios-Jaraquemada JM. Conservative vs. radical management of placenta accreta spectrum (PAS). Curr Obstet Gynecol Rep. 2020:9(12)1-8.

13. Peng $X$ et al. Parallel transverse uterine incisions, a novel approach for managing heavy hemorrhage and preserving the uterus: a retrospective cohort study for patients with anterior placenta previa and accreta. Medicine. 2019;98(44):e17742.

14. Palacios-Jaraquemada JM et al. Anterior placenta percreta: surgical approach, hemostasis and uterine repair. Acta Obstet Gynecol Scand. 2004;83(8):738-44. 